\title{
OS TRANSTORNOS \\ DEPRESSIVOS: um olhar sobre a reestruturação dos cuidados em saúde mental
}

DOI: 10.22289/2446-922X.V4N3A9

\author{
Leidiane Aparecida de Oliveira ${ }^{1}$ \\ Vania Cristine de Oliveira
}

\section{RESUMO}

Os transtornos depressivos vêm acompanhando o ser humano ao longo da sua existência. São distúrbios afetivos responsáveis pela regulação do humor. Em razão da alta incidência em decorrência dos impactos causados na funcionalidade do portador, este estudo teve como objetivo verificar estratégias, que possam proporcionar ações diretivas vinculadas à promoção da saúde em função de promover e edificar novas ações que visam possibilitar uma melhor qualidade de vida, associando aos modelos de saúde metal. Foi realizada uma revisão conceitual da literatura, sendo desenvolvidos alguns critérios de busca nas bases de dados Scielo, Pepsic, Google Acadêmico. Na busca pelos artigos foram anexados os descritores como depressão; estratégias; psicologia; promoção; prevenção. Optou-se por textos nacionais e internacionais americanos e espanhóis publicados entre 2000 a 2017, visando estudos pertinentes que dialogassem com os conhecimentos brasileiros. Os estudos apontam à importância das criações de estratégias dentro do campo da saúde mental, salientando suas contribuições vinculadas a promoção de saúde na amenização dos sofrimentos mentais, transformando tanto a percepção do portador acometido pelo adoecimento como modificando o ambiente onde se encontra inserido dentro das redes de atenção Básica, através da humanização dos cuidados. A Promoção da Saúde renasce dos paradigmas não como teoria nova, mas como estratégias se apoiando em autonomia, responsabilidade e justiça desmistificando o saber.

Palavras-chave: Depressão; Estratégias; Psicologia; Promoção; Prevenção.

\section{ABSTRACT}

Depressive disorders have been following the human being throughout his life. They are affective disorders responsible for mood regulation. Because of the high incidence due to the impacts caused on the functionality of the bearer, this study had as objective to verify strategies that can provide directives related to the promotion of health in function of promoting and building new actions that aim to enable a better quality of life, associating with the models of mental health. A conceptual review of the literature was carried out, and some search criteria were developed in the Scielo, Pepsic, and Academic data bases. In the search for the articles were appended the descriptors depression; strategies; psychology. promotion. prevention. National and international,

\footnotetext{
${ }^{1}$ Endereço eletrônico de contato: leidianeapoliveira1@hotmail.com

Recebido em 20/09/2018. Aprovado pelo conselho editorial para publicação em 16/11/2018.
}

Rev. Psicol Saúde e Debate. Dez., 2018:4(3):96-109. 
American and Spanish texts were published between 2000 and 2017, aiming at pertinent studies that dialogue with the Brazilian knowledge. The studies point out the importance of the creation of strategies within the field of mental health, emphasizing their contributions linked to health promotion in the alleviation of mental suffering, transforming both the perception of the sufferer affected by illness and modifying the environment where it is inserted within the networks of basic care through the humanization of care. Promotion is born of paradigms not as a new theory, but as strategy that rely on autonomy, responsibility and justice, demystifying knowledge.

Keywords: Depression; Strategies; Psychology; Promotion; Prevention.

\section{INTRODUÇÃO}

Em tempos modernos onde a ciência se faz presente no campo da saúde mental, os transtornos depressivos são altamente prevalentes em todo o mundo. Considerado um transtorno mental, os transtornos depressivos chegam ao ápice das discussões em todo o mundo, sendo considerada a segunda doença mais prevalente nos meios sociais e de saúde mental, podendo se manifestar em qualquer idade e em qualquer fase da vida, em cursos de episódios ou crônicos.

Através das notas divulgadas nos relatórios emitidos pela Organização Mundial de Saúde (OMS, 2017), são reforçadas campanhas em prol de amenizar os sofrimentos psíquicos de ordem emocional e comportamental, como forma de prevenir seu crescimento. Neste sentido, a OMS vem noticiando informações que servem como alertas perante o aumento global dos transtornos depressivos, sendo considerada uma doença silenciosa e incapacitante, tendo como consequências atos suicidas com comprovação de morte.

O Ministério da Saúde (Brasil, 2008) aborda, através da obra 'Saúde Mental e Atenção Básica: o vínculo e diálogo necessários', ações relacionadas à criação de vínculos através dos diálogos estabelecendo um acolhimento dentro da atenção básica, viabilizando o apoio matricial, através dos suportes em ações e projetos, como forma de avançar com novas propostas, definindo novas concepções nas práticas em saúde.

Baseado na Organização Mundial de Saúde (OMS, 2010) os critérios para os atendimentos a pacientes com transtornos depressivos estão consolidados ao atendimento em Centro de Atenção Psicossocial (CAPS), em um modelo sustentado pelas políticas de ação que visam à atenção ao atendimento integral do individuo.

Conforme Jorge et al. (2011) os novos paradigmas em saúde mental vêm rompendo com modelos biomédicos, pois durante muitos anos os tratamentos consistiam na exclusão e no modelo asilar, em que os pacientes com transtornos se viam exclusos, com violação dos seus

Rev. Psicol Saúde e Debate. Dez., 2018:4(3):96-109. 
direitos. Através da reforma psiquiátrica foram surgindo novas versões e visões sobre tratamentos de pacientes com transtornos psiquiátricos, o que mais tarde vieram consolidar as políticas de saúde pautadas no processo de desinstitucionalização, evidenciando a assistência integral e resolutiva.

O campo da psicologia vista como ciência e profissão não vêm abordar a depressão somente como transtorno mental vinculado à incapacitação do sujeito adoecido, mas surge com visão de promoção à saúde como forma de edificar e de se fazer saúde. Através de iniciativas políticas, científicas e sociais levantam-se estratégias voltadas para humanização do cuidado, pautado na promoção e prevenção em prol do bem-estar mental dos usuários (Jorge et al., 2011).

As políticas de atenção à saúde mental associada à psicologia representam uma ferramenta do modelo positivista, que aderem à integralidade e humanização reduzindo a unidimensionalidade, rompendo com os modelos tradicionais. Dentro dos parâmetros da literatura, o manejo das estratégias associadas às práticas vinculadas à saúde mental no atendimento e acolhimento dos indivíduos portadores dos transtornos depressivos, na ótica da promoção a saúde, é pouco explorada, fazendo necessárias novas pesquisas, que viabilizem inovações dentro das políticas em saúde mental (Branco \& Bosi 2014).

A pesquisa se fundamenta em revisão conceitual da literatura de cunho exploratório, embasada em estudos qualitativos, destacando a inclusão das ações em saúde mental dentro da atenção básica, norteadora dos novos modelos assistenciais, evidenciando um olhar mais humanizado sobre os cuidados com os pacientes depressivos. Em razão da alta incidência em decorrência dos impactos causados na funcionalidade do portador. A pesquisa visa verificar estratégias, que possam proporcionar ações diretivas vinculadas à promoção da saúde em função de promover e edificar novas ações que visam possibilitar uma melhor qualidade de vida, associando aos modelos de saúde mental.

\section{AS CONTEXTUALIZAÇÕES DOS TRANSTORNOS DEPRESSIVOS: fatores predisponentes e diagnóstico diferencial}

A conceituação da depressão é vista como uma variedade de perturbações psicopatológicas, o que diferencia quanto aos sintomas, curso, gravidade e prognóstico. Constantemente confundem estados de estresse e de tristeza como depressão, sendo a depressão muito mais intensa e dolorosa, o que acarreta a imobilidade funcional na vida do portador (Souza et al., 2001 citado por Peron, Neves, Brandão, \& Vicentini, 2004, p. 45).

Rev. Psicol Saúde e Debate. Dez., 2018:4(3):96-109. 
Segundo descrito por Molina et al. (2012) na atualidade os transtornos depressivos são altamente prevalentes nos campos psíquicos sendo responsáveis pelos impactos na vida dos portadores e seus familiares. A depressão lidera os rankings das doenças cuja inabilidade social se apresenta com norteadora do aumento das mortes entre jovens de 18 a 44 anos de idade.

De acordo Motta, Moré e Nunes (2017) as ações desenvolvidas para trabalhar com transtornos depressivos, referem-se às estruturas organizacionais, as quais promovem procedimentos envolvendo atendimentos profissionais, vinculados às discussões sobre o manejo dos transtornos depressivos, cujos quadros clínicos passam por alterações sintomatológicas, aumentando sua gravidade.

Arantes (2007) menciona em seus estudos sobre a depressão, percebe-se um contexto de negligência por parte do portador da patologia que diante dos diagnósticos não segue os tratamentos, sendo em muitos dos casos relatados desvinculação das redes básicas de saúde, ocasionando um aumento dos riscos de vir acometer um suicídio.

Inúmeros estudos vêm sendo elaborados sobre a etiologia para se obter o resultado da origem das causas da depressão. O que se tem descoberto através das investigações é que não há uma causa definida, mas sim fatores predisponentes que contribuem para a manifestação dos transtornos. Fatores relacionados à genética com os quadros depressivos são significativos na evolução do transtorno depressivo, porém não há evidências quanto à ocorrência do desencadear no organismo. Os estudos referem-se à herança genética relacionada ao parentesco de primeiro grau e gêmeos monozigóticos, aqueles que possuem o mesmo código genético, podendo obter mais chances para o desenvolvimento da patologia, o que não resulta como prova concreta da sua ocorrência (Ferreira, 2011).

Diante de investigações, alguns psicólogos afirmam que os ambientes familiares adoecido com pais rígidos, exigentes, de caráter severo podem estar relacionados aos fracassos em alguma etapa da vida dos filhos, propiciando o desencadeamento de sinais de vulnerabilidade, apresentando a propensão ao desenvolvimento da depressão. Outro aspecto pode estar relacionado com pouco afeto materno ou ausência da afetividade, porém não existem evidências científicas comprovadas (Grevet, Cordioli, \& Fleck, 2005).

Outros fatores estão relacionados à personalidade, visto que os seres humanos estão propensos a desencadear pico de depressão, tornando-se deprimidos em qualquer fase da vida em diferentes circunstâncias, porém pessoas com obsessões, fanatismo religioso, com comportamentos rígidos e com variações de humor frequente e continuada, têm uma predisposição maior para o surgimento da patologia (Sousa, Pinho, \& Garcia, 2016). 
Segundo Jardim (2011) a ocorrência de uma experiência traumática, falecimento de ente querido, termino de um relacionamento, doença grave etc., podem estar associados aos estados emocionais adoecidos sendo um fator precursor para desencadeamento da patologia, pois decorre das alterações da percepção do indivíduo sobre os fatos, ocasionando o rebaixamento do humor.

De acordo com Joca, Padovan e Guimarães (2003), os transtornos depressivos podem estar associados aos neurotransmissores, sendo responsáveis pelas variações de humor no organismo, obtendo como consequências alterações nos estados emocionais e o comprometimento da funcionalidade do indivíduo, essas alterações são amenizadas por medicamentos conhecidos como antidepressivos, que agem produzindo um aumento dos fatores neuroquímicos relacionados à serotonina e noradrenalina nos espaços entre os neurônios.

O termo diagnóstico origina-se da palavra Diagnose, vinculada às ações de promover a identificação das causas e o desenvolvimento da patologia, através da avaliação e análise do prognóstico, os quais proporcionam mais informações a respeito da patologia desencadeada, assim podendo ser classificada em várias escalas de categorias como leves moderadas ou graves (Baroni, Vargas, \& Caponi, 2010).

Com base nos estudos deferidos pelo Manual Diagnósticos e Estáticos dos Transtornos Mentais (DSM-5), os transtornos depressivos são denominados como Transtorno Desruptivo da Desregulação do Humor, que significa desestabilizar os estados afetivos, modificando e alternando sua disposição e ânimo, obtendo como característica diagnóstica a presença constante de humor triste, vazio ou irritabilidade, acompanhado de alterações físicas e cognitivas que resultam na incapacitação do ser humano de realizar atividades com exatidão e compromisso (Associação Americana de Psiquiatria [APA], 2014).

As escalas de classificação se dão através das identificações dos sintomas, intensidade e duração, sendo analisada sua qualidade e quantidade. Essas escalas decorrem das áreas de psiquiatria e psicologia, as quais investigam os sintomas e os critérios necessários para o diagnóstico, podendo evoluir e se agravar. Dentro da classificação dos transtornos mentais e sua influência é necessário identificar as variáveis que atuam na produção do sofrimento do portador (Bahls \& Bahls, 2002).

A dificuldade enfrentada no diagnóstico dos transtornos depressivos refere-se à presença de patologias que se associam dentro dos quadros clínicos, dificultando identificação dos mesmos. No entanto pode estar relacionada à camuflagem entre os sintomas e às negligencias diante da falta de atenção, colaborando para que passem despercebidos alguns fatores anormais que possam contribuir para agravamento do quadro (Molina et al., 2012).

Rev. Psicol Saúde e Debate. Dez., 2018:4(3):96-109. 
Como explica Cunha (2000) o Psicodiagnóstico é um método que tem como finalidade identificar o diagnóstico diferencial, englobando os sintomas, dados clínicos, observações dos comportamentos durante todo o processo e os resultados dos testes aplicados, cuja função é determinar o diagnóstico através da classificação da patologia e do uso dos manuais de saúde mental. O Psicodiagnóstico pode ajudar a ilustrar os resultados coletados colaborando para uma avaliação mais criteriosa.

Dentro das avaliações psicológicas, os inventários da depressão (BDI-II) são os mais utilizados por psicólogos que visa medira intensidade que o transtorno afeta o indivíduo, auxiliando no processo investigatório do diagnóstico, fornecendo dados sobre a gravidade do quadro e a qualidades dos pensamentos. Obtendo uma escala de correção que varia de acordo de com a pontuação podendo ser aplicado nas faixas etária dos 13 anos até meados da terceira idade (Ely, Nunes, \& Carvalho, 2014).

\section{OS DESAFIOS NO CAMPO DA SAÚDE MENTAL NUM PROCESSO DE CONSTRuÇÃo ATRAVÉS DA PROMOÇÃo E PREVENÇÃo NA HUMANIZAÇÃO DOS CUIDADOS}

As políticas de saúde mental estão sendo amparadas pelos mecanismos do saber, que vêm estudar e transformar os desafios que antes eram negligenciados, e que hoje são revertidos em estratégias de promoção e prevenção. Segundo Perrusi (2015) a depressão é um dos transtornos que tem como característica o sofrimento psíquico, visto com uma patologia desafiadora pelos profissionais que atuam na saúde, retratam a dificuldade de diagnosticar a doença, o que demanda ações para formular o manejo do tratamento com utilização dos medicamentos.

A problematização envolvendo os transtornos depressivos, configurados como 'doença do século', abrange aspectos de negação e aceitação. Infelizmente existe uma aceitação maior da doença física, aquela que agride o corpo de forma visível e identificável através de exames clínicos, sendo diagnosticada e melhor compreendida. Contudo, os transtornos depressivos são desordens metais e emocionais que acarretam uma dificuldade na desmistificação dos sintomas. Portanto a patologia sendo mais bem esclarecida tende a diminuir seus impactos em torno da vida do portador (Arantes, 2007).

Dentro das políticas de saúde mental, os obstáculos relacionados à ampliação das redes de atendimentos vinculados aos cuidados na atenção básica dentro dos espaços territoriais, Rev. Psicol Saúde e Debate. Dez., 2018:4(3):96-109. 
permeiam ações que visam à compreensão dos profissionais em lidar com a expansão dos transtornos mentais, trabalhando a reinserção do portador na comunidade para que o mesmo não seja excluído nem constrangido socialmente (Moliner \& Lopes, 2013).

Segundo Reinaldo (2008) a partir do ano de 1960 surgiu uma nova concepção de tratamento dos transtornos mentais, através dos novos modelos relacionados à psiquiatria comunitária, que consistia na reorganização das políticas diretivas em saúde mental. A implantação das políticas nacionais consiste na redução dos leitos hospitalares como internações permanentes, dando atenção aos cuidados assistenciais ao portador, priorizando a reestruturação nos serviços e ações dentro dos centros de atendimentos (Brasil, 2008).

Através das implantações dos modelos de assistências em saúde surge a implantação da Política Nacional de Promoção a Saúde, decretada pela portaria MS/GM n 687, de 30 de março de 2006, sendo propostas inovações na forma de planejamento, ações, análise e avaliação dos serviços de saúde. Essas ações estão vinculadas à atenção básica, formando conjuntos de atitudes individuais ou coletivas que possam contribuir para ascensão da proteção à saúde, associando à prevenção de patologias e seus respectivos fatores de agravamento e à diminuição dos dados estatísticos, o diagnóstico, tratamentos e reabilitação (Brixner et al., 2017).

A promoção da saúde é mais bem definida como processo de potencializar o indivíduo a ter maior consciência sobre as melhorias relacionadas à manutenção de sua saúde, porém este conceito se fundamenta através dos recursos sociais e pessoais promovendo a capacitação física. Sendo assim, a promoção não se restringe apenas à responsabilidade dos setores da saúde, mas à junção do paciente e seu estilo de vida com os cuidados deliberados nas redes de saúde, na busca pelo bem estar (OMS, 2010).

De acordo com Cordeiro, Oliveira, Melzer, Ribeiro e Rigonatti (2010) a prevenção é um conjunto de ações antecipatórias realizadas por profissionais de saúde com responsabilização, a fim de promover as decisões técnicas como ações diretivas e educativas visando à ascensão, conservação da saúde e a diminuição da evolução da doença através da interceptação.

Dentro da esfera da saúde mental, as ações preventivas e ações assistenciais estão associadas ao ato de cuidar com redução e o alivio dos sofrimentos deferidos pelos preceitos de equidade e Justiça social (Norman, 2013).

A prevenção está relacionada às ações elaboradas frente aos fatores predisponentes sociais e ambientais, ou já com a ocorrência da patologia instalada, sendo subdividida entre fases distintas. A primeira fase é a prevenção primária, que se dá com a ocorrência nos períodos antecessores à doença, conhecido também como pré-patogênicos que envolvem a promoção da saúde associada à proteção contra uma determinada patologia emergente. A prevenção Rev. Psicol Saúde e Debate. Dez., 2018:4(3):96-109. 
secundária ocorre já com a patologia instalada, sendo inserido o diagnóstico, tratamento extemporâneo, bem como a limitação, a incapacidade física e mental. A prevenção terciária consiste seus trabalhos em evitara incapacitação do sujeito frente à invalidez, através das ações dentro do processo destinado à reabilitação do sujeito (Cordeiro et al., 2010).

\section{AS CONTRIBUIÇÕES DA PSICOLOGIA ACERCA DAS ESTRATÉGIAS DO MANEJO DOS TRANSTORNOS DEPRESSIVOS}

A psicologia no âmbito da saúde mental se fundamenta na construção e na aplicabilidade dos seus estudos, através das técnicas psicológicas usadas em prol da compreensão da doença e dos cuidados para manutenção do bem estar, possibilitando uma melhor qualidade de vida, através de ações desenvolvidas no campo da saúde mental (Lemos \& Silva, 2009).

Frente ao aumento dos transtornos depressivos na atualidade, Lemos e Silva (2009) traz uma discussão pertinente que recai sobre as ações desenvolvidas dentro da atuação do psicólogo frente ao adoecimento mental, correlacionado ao manejo dos pacientes acometidos pelos transtornos.

Os psicólogos no exercício da sua profissão trabalham com os atendimentos psicológicos que visam acessar a subjetividade do usuário, a fim de compreender o sofrimento mental ocasionado pela patologia. Ao lidar com os transtornos depressivos, a escuta é uma ferramenta primordial no processo de identificação dos fatores ocultos e da própria demanda do portador na compreensão da sua vivência (Motta et al., 2017).

De acordo com APA (2004), as intervenções praticadas no campo da saúde mental pelos psicólogos visam oferecer melhoria na qualidade de vida ofertando serviços qualificados, com o objetivo de diminuir os casos enfermos em fases de internamentos, reduzindo a utilização de medicamentos em prol dos cuidados com os indivíduos favorecendo a aplicação de recursos mais adequados a cada caso.

Os atendimentos psicológicos se configuram em um processo de construção de autonomia do indivíduo, potencializando o reconhecimento de sua condição atual de adoecimento, como se configuram os transtornos no organismo, viabilizando o diálogo como forma de tratamento, sendo um processo que envolve a psicoeducação com a finalidade de diminuir os sintomas associados à prevenção de novas fases dentro dos quadros clínicos (Motta et al., 2017).

Rev. Psicol Saúde e Debate. Dez., 2018:4(3):96-109. 
Segundo Ladeia e Soares (2013) os atendimentos são pautados em individuais e grupais. Sendo que os individuais são precursores de ações e atenção particulares direcionadas às intervenções focadas na patologia, acompanhando o paciente passo a passo a partir das suas próprias elaborações frente ao seu sofrimento. Já os atendimentos grupais se fundamentam como estratégias terapêuticas, que visam à construção de vinculo e de apoio fortalecendo as relações sociais, que tem com o propósito de trabalhar as trocas de experiências entre os envolvidos, transformando sua subjetividade através das identificações, fatos e histórias semelhantes a outros indivíduos.

O atendimento psicoterapêutico individual abrange a construção de uma aliança entre o paciente e o psicólogo através da fala, buscando dar sentido a suas angustias frente ao seu sofrimento visando compreender os multi-fatores do adoecimento e de sua dimensão dentro dos aspectos psicológicos. Essa tarefa e muito importante, pois trabalha a ressignificação na sua forma ver e de viver, dando novos sentidos a sua vida (Motta et al., 2017).

De acordo com Jorge et al. (2011) os atendimentos em grupo são pautados semanalmente, como forma do indivíduo vivenciar experiências novas dentro de um grupo como histórias em comum, obtendo o objetivo de resgatar a capacidade de se socializar com outros participantes. Sendo demonstradas em rodas de conversas, dinâmicas, encenações e dramatizações etc., as atividades terapêuticas proporcionam ao indivíduo, vivenciar os sentimentos de universalidade, suporte, compreensão, encorajamento e fortalecimento para conhecer a si mesmo.

Dentro da dimensão psicossocial, as estratégias estão voltadas para os tratamentos dos transtornos depressivos como recurso complementares vinculados as oficinas terapêuticas, que obtém de forma diferencial a sua escuta. Sendo oficinas voltadas para pintura, criações de obras de artes com argila, desenho, dança teatro e música que visa à reabilitação associada ao lazer e o bem-estar. Recursos estes que estão pautados no reconhecimento das possibilidades dos usuários referente ao seu tratamento (Sampaio \& Garcia, 2015).

Conforme OMS (2010) outras intervenções estão relacionadas aos cuidados e atendimentos a portadores dos transtornos depressivos fora dos contextos institucionais, em ambientes diversificados e estratégicos, sendo desenvolvidas pelo acompanhamento terapêutico (AT), o qual procura observar e analisar os aspectos adoecidos do paciente, procurando formas de transformar o cotidiano do sujeito a fim de proporcionar uma melhor qualidade de vida. Estrategicamente a criação de bom vínculo com o paciente e seus respectivos familiares, fazendo de forma empática e com compromisso, são ferramenta cruciais para amenizar os impactos e os riscos de suicídios. 
Segundo Motta et al. (2017) em algumas Unidades Básicas de Saúde (UBS) vinculadas ao Núcleo de Apoio a Saúde da Família (NASF), existem ainda outros grupos de auto ajuda, os quais promovem na prática exercícios físicos, atividades de relaxamentos, orientações sobre alimentação, palestras, entre outros, cujo os benefícios para os pacientes com transtornos depressivo se articulam entre bem estar físico e psicológico, proporcionando a ativação comportamental com o fortalecimento da autoestima e dos relacionamentos sociais e pessoais.

As estratégias de cuidados a portadores dos transtornos depressivos se norteiam sobre os cuidados dos indivíduos, vinculados ao acolhimento, consultas de saúde mental, encaminhamento ao médico da família ou psiquiatra, grupos de apoio (Daré \& Caponi, 2016).

O processo de medicalização do usuário é um recurso no tratamento dos quadros considerados graves, os quais são necessários à utilização dos antidepressivos, que agem no organismo estabilizando as oscilações de humor. Sendo medicamentos desenvolvidos para manutenção do equilíbrio do portador diante do seu sofrimento mental (Daré \& Caponi, 2016).

Entre as estratégias utilizadas no âmbito do assistencialismo na atenção básica, segundo Cordeiro et al. (2010) as propostas associadas à prevenção contribuem para o cuidado e desmistificação dos quadros através das informações antecipatórias estabelecendo os parâmetros de políticas assistenciais de saúde vinculados às campanhas, visando a diminuição dos estigmas sociais relacionados ao transtorno mental, sendo a disseminação de orientação à sociedade proporcionando o fortalecimento para aceitabilidade dos tratamentos.

Dentro do contexto de saúde mental, a psicologia contribui em planejamento de ações vinculadas à promoção e prevenção, focalizada na informação e reorganização das práticas em prol da acessibilidade dos usuários e o progresso comunitário. Sendo direcionado a consultas psicológicas com adesão clínica associada ao médico da família buscando avaliações e posteriormente podendo ser encaminhado para atendimentos psiquiátricos em casos que identifiquem um agravamentos dos quadros. Porém, as praticas direcionadas aos cuidados continuados se configuram em atendimentos em domicílio em casos onde o paciente esteja debilitado, com fator de dependência e vulnerabilidade. Portanto a Psicologia não se restringe somente a estudar os eventos mantedores de seus sofrimentos, mas o sujeito como um todo e as suas condições e dimensões (Teixeira, 2004).

\section{CONCLUSÂO}

Rev. Psicol Saúde e Debate. Dez., 2018:4(3):96-109. 
Os transtornos depressivos são vistos atualmente como um dos grandes vilões pela forma silenciosa e desafiadora que vem se apresentando nos meios sociais. Desafios estes que são vivenciados pelos profissionais de saúde na busca pela aceitabilidade ao tratamento e disponibilização de recursos para criação de novos modelos de tratamento, os quais possam amenizar o sofrimento do portador. A psicologia atrelada às políticas de saúde mental vem rompendo com os modelos pré-estabelecidos de 'saúde-doença', transformando o olhar através da promoção e prevenção, enfatizando critérios como atenção, articulações de ações, recuperação e reabilitação social.

Ressalta-se a importância da psicologia como ciência e profissão nos tratamentos dos portadores dos transtornos depressivos, pois se acredita na coprodução do indivíduo e de seu contexto, desenvolvendo através dos vínculos estabelecidos as possibilidades de construção da autonomia e bem estar dos usuários.

Os estudos encontrados apontam para a importância da criação de projetos enviesados na estruturação de estratégias correlacionados com a promoção da saúde, sendo o acolhimento com a criação de vínculo entre usuário e o profissional de saúde, a formação de grupos de apoios, com indivíduos que estejam passando pelos mesmos problemas, onde possam fazer as trocas de experiências, com a finalidade de se ajudar mutuamente, trabalhando o fortalecimento do grupo. Através das consultas clínicas psicológicas voltadas para psicoterapia, enfatizando o acompanhamento do paciente em seu tratamento, fazendo intervenções necessárias que possam contribuir para uma melhor aceitabilidade dos tratamentos por parte do individuo, visando à reabilitação do sujeito acometido pela doença. A partir da construção do trabalho de assistência aos cuidados dos usuários que serão direcionados ao médico da família com propósito de obter um acompanhamento sobre a medicação em caso mais graves com alterações fisiológicas e cognitivas.

As contribuições das ações desenvolvidas para trabalhar com indivíduos depressivos são altamente benéficas para estabilização do quadro, frente às oscilações emocionais e comportamentais.

Embora exista uma gama de estudos científicos elaborados para tentar achar uma explicação plausível sobre natureza dos transtornos, há poucos estudos abordando o manejo de estratégias na adesão dos cuidados a portadores.

A literatura ainda aponta a necessidade de novos estudos sobre os transtornos depressivos e seus critérios de diagnóstico, sabendo que a patologia não tem uma cura definitiva, contudo, existe a possibilidade de estabilização do quadro, ofertando uma melhor qualidade ao portador.

Rev. Psicol Saúde e Debate. Dez., 2018:4(3):96-109. 


\section{REFERÊNCIAS}

Arantes, V. (2007). Depressão na atenção primária á saúde. Rev. Bras. Med. Fam. e Com., 2(8), 261-270.

Associação Americana de Psiquiatria (2004). Psychology: Promoting Health and Well-Being trough High Quality, Cost-Effective Treatment, Estados Unidos: Autor.

Bahls, S. C., \& Bahls, C. R. (2002). Depressão na adolescência: características clínicas. Interação em Psicologia, 6(1), 49-57.

Baroni, M. P., Vargas, S. F., \& Caponi, S. N. (2010). Diagnóstico como nome próprio. Psicologia \& Sociedade, 22(1), 70-77.

Branco, A. B. A. C., \& Bosi, M. L. M. (2014) Integralidade e humanização em saúde mental: experiências de usuários em dois centros de atenção psicossocial. Cad. Saúde Colet., 22 (2), 221-222.

Brasil (2008.). Ministério da Saúde. Secretaria de Atenção à Saúde Departamento de Ações Programáticas Estratégicas, Departamento de Atenção Básica Coordenação Geral de Saúde Mental, Coordenação de Gestão da Atenção Básica. Saúde mental e atenção básica o vínculo e o diálogo necessários inclusão das ações de saúde mental na atenção básica. Brasília, DF: Autor.

Brixner, B., Muniz, C., Renner, J. D. P., Pohl, H. H., Garcia, E. L., \& Krug, S. B. F. (2017). Ações de promoção da saúde nas estratégias saúde da família. Revista do Departamento de Educação Física e Saúde Cinergis, 18(1), 386-390.

Cordeiro, Q., Oliveira, A. M., Melzer, D., Ribeiro, R. B., \& Rigonatti, S. P. (2010). Prevenção em saúde mental. Revista do Curso de Direito da Faculdade de Humanidades e Direito, 7(7), 39-53.

Cunha, A. (2003). Psicodiagnóstico V. (5a ed.). São Paulo: Artmed.

Daré, K., \& Caponi, S. N. (2016). Cuidado ao indivíduo com depressão na atenção primária em saúde. ECOS: Estudos Contemporâneos da Subjetividade, 7(1), 12-22.

Ely, P., Nunes, M. F. O., \& Carvalho, L. F. (2014) Avaliação psicológica da depressão: levantamento de testes expressivos e autorrelato no Brasil. Avaliação Psicológica, 13(3), 419-426.

Ferreira, F. M. S. (2011). A criança com depressão. Dissertação de Mestrado em Ciências da Educação-Educação Especial, Escola Superior de Educação Almeida Garrett, Departamento de Ciências da Educação, Lisboa, Portugal, 2011.

Grevet, E. H., Cordioli, V., \& Fleck, A. (2005). Depressão maior e distimia: diretrizes e algoritmo para o tratamento farmacológico. Porto Alegre: Artmed.

Rev. Psicol Saúde e Debate. Dez., 2018:4(3):96-109. 
Jardim, S. (2011). Depressão e trabalho: ruptura de laço social. Rev. bras. Saúde ocup, 36(123), 84-92.

Joca, S. R. L., Padovan, C. M., \& Guimarães, F. S. (2003). Estresse, depressão e hipocampo. Rev. Bras. Psiquiatr., 25(Supl II), 46-51.

Jorge, M. S. B., Pinto, D. M., Quinderé, P. H. D., Pinto, A. G. A., Sousa, F. S. P., \& Cavalcante, C. M. (2011). Promoção da saúde mental-tecnologias do cuidado: vínculo, acolhimento, coresponsabilização e autonomia. Ciência \& Saúde Coletiva, 16(7), 3051-3060.

Ladeia, K. D., \& Soares, M. L. B. A. (2013). Estudo analítico-comparativo: psicoterapia de grupo e psicoterapia individual a partir da perspectiva do sujeito. Trabalho de Conclusão de Curso Graduação em Psicologia, Centro Universitário Católico Salesiano Auxilium, Lins, SP.

Lemos, M. C. Jr., \& Silva, F. (2009). Psicologia de orientação positiva: uma proposta de intervenção. Ciência \& Saúde Coletiva, 14(1), 233-242.

Molina, L. R., Wiener, D., Branco, J. C., Jansen, K., Souza, M. D., \& Tomasi, E. et al. (2012). Prevalência de depressão em usuários de unidades de atenção primária. Rev. Psiq. Clín., 39(6), 194-197.

Moliner, J., \& Lopes, B. M. (2013). Saúde mental na atenção básica: possibilidades para uma prática voltada para a ampliação e integralidade da saúde mental. Saúde Soc., 22(4), 10721083.

Motta, L. C., Moré, O. L., \& Nunes, S. H. (2017). O atendimento psicológico ao paciente com diagnóstico de depressão na Atenção Básica. Ciência \& Saúde Coletiva, 22(3), 911-920.

Norman, A. H. (2013). Promoção da saúde: um desafio para a atenção primária. Rev. Bras. Med. Fam. Comunidade, 8(28), 153- 154.

Organização Mundial de Saúde (2010). Manual mhGAP de intervenções para transtornos mentais, neurológicos e por uso de álcool e outras drogas para a rede de atenção básica à saúde (J. M. Bertolote, Trad.). (p. 120). (S. I.): Autor.

Peron, A. P., Neves, G. Y. S., Brandão, M., \& Vicentini. V. E. P. (2004). Aspectos biológicos e sociais da depressão. Arq. Ciênc. Saúde Unipar, 8(1), 45-48.

Perrusi, A. (2015). Sofrimento psíquico, individualismo e uso de psicotrópicos: saúde mental e individualidade contemporânea. Revista de Sociologia da USP, 27(1), 139-159.

Reinaldo, S. M. (2008). Saúde mental na atenção básica como processo histórico de evolução da psiquiatria comunitária. Rev. Enferm. Esc. Anna Nery, 12(1), 173-178.

Sampaio, J. J. C., Garcia, C. Filho. (2015). Saúde Mental: política, trabalho e cuidado (1a ed.). Fortaleza, CE: UECE.

Sousa, L. B., Pinho, B. S., \& Garcia, R. F. (2016). Transtorno Depressivo. In M. S. Bessa, J. C. Filho Garcia, \& D. Q. C. Rocha (Orgs.). Liga acadêmica de psiquiatria e saúde mental:

Rev. Psicol Saúde e Debate. Dez., 2018:4(3):96-109. 
concepções e marcos conceituais na produção do conhecimento. (1a ed., Cap. 4, pp. 7595). Fortaleza, CE: EdUECE.

Teixeira, C. A. (2004). Psicologia da Saúde. Análise Psicológica, 22(3), 441-448.

Rev. Psicol Saúde e Debate. Dez., 2018:4(3):96-109. 